\title{
Simulation of the Combined Effects of Dipole Emitter Orientation, Mie Scatterers, and Pillow Lenses on the Outcoupling Efficiency of an OLED
}

\author{
Ju Seob Lee ${ }^{1}$, Jong Wan Lee ${ }^{1}$, Jaehoon Park $^{2}$, and Jae-Hyeon $\mathrm{Ko}^{1 \dagger}$ \\ ${ }^{1}$ Department of Physics, Hallym University, Hallydaehakgil 39, Chuncheon 200-702, Korea \\ ${ }^{2}$ Department of Electronic Engineering, Hallym University, Hallydaehakgil 39, Chuncheon 200-702, Korea
}

(Received June 19, 2014; Revised manuscript July 16, 2014; Accepted July 17, 2014)

\begin{abstract}
The net effect of the emitter orientation, Mie scatters, and pillow lenses on the outcoupling efficiency (OCE) of a bottomemitting OLED having an internal photonic crystal layer was investigated by a combined optical simulation based on the finite-difference time-domain method (FDTD) and the ray-tracing technique. The simulation showed that when the emitter orientation was horizontal with respect to the OLED surface, the OCE could be increased by $54 \%$ when a photonic crystal layer was employed, while it could be improved by $86 \%$ under optimized conditions of Mie scatters and pillow lenses applied to the glass substrate. The peculiar intensity distribution of the OLED, caused by the periodic lattice structure of the photonic crystal layer, could be ameliorated by inserting Mie scatters into the glass substrate. This study suggests that conventional outcoupling structures combined with control of the emitter orientation could improve the OCE substantially.
\end{abstract}

Keywords: OLED, Outcoupling efficiency, Emitter orientation

OCIS codes: (110.2945) Illumination design; (120.5240) Photometry; (230.3670) Light-emitting diodes

\section{쌍극자 광원의 진동방향, Mie 산란자, 그리고 Pillow 렌즈가 OLED의 광추출효율에 미치는 영향에 대한 시뮬레이션 연구 \\ 이주섭 ${ }^{1} \cdot$ 이종완 $^{1} \cdot$ 박재훈 $^{2} \cdot$ 고재현 $^{1 \dagger}$ \\ ${ }^{1}$ 한림대학교 전자물리학과 \\ (ㅇ) 200-702 강원도 춘천시 옥천동 한림대학길 39 \\ 2한림대학교 전자공학과 \\ (ㅇ) 200-702 강원도 춘천시 옥천동 한림대학길 39}

(2014년 6월 19일 받음, 2014년 7월 16일 수정본 받음, 2014년 7월 17일 게재 확정)

\begin{abstract}
본 연구에서는 FDTD와 광선추적기법을 결합한 시뮬레이션을 활용해 광원으로 설정된 쌍극자의 진동 방향, 유리기판에 적용된 $\mathrm{Mie}$ 산란입자와 Pillow 렌즈가 광결정 구조가 포함된 OLED의 광추출효율에 미치는 영향을 조사하였다. 쌍극자 광원의 진동방향 이 OLED 표면에 대해 수평인 경우, 광결정구조만 적용된 OLED의 효율이 $54 \%$ 인데 반해 최적화된 조건의 Mie 산란입자, Pillow 렌즈가 적용된 $\mathrm{OLED}$ 는 약 $86 \%$ 의 광추출효율을 나타냈다. 아울러 광결정 구조로 인해 특정 각도로 광도가 증가하는 문제점이 $\mathrm{Mie}$ 산란입자의 산란효과로 인해 완화될 수 있음을 알았다. 본 연구는 광추출효율을 향상시키는 다양한 광학구조를 적용함과 더불어 발광체 유기분자의 배향을 조절함으로써 OLED의 효율을 큰 폭으로 향상시킬 수 있음을 보여준다.
\end{abstract}

Keywords: 유기발광다이오드, 광추출효율, 발광자 방향

OCIS codes: (110.2945) Illumination design; (120.5240) Photometry; (230.3670) Light-emitting diodes

${ }^{\dagger}$ E-mail: hwangko@hallym.ac.kr

Color versions of one or more of the figures in this paper are available online. 


\section{I. 서 론}

유기발광다이오드(Organic Light Emitting Diode, OLED)의 응용 분야는 최근 휴대폰이나 모바일용 디스플레이를 넘어 서 대형 TV와 일반 조명 분야로까지 확대되고 있다. OLED 의 적용 분야가 넓어지는 만큼 OLED의 성능을 향상시키기 위한 다양한 연구가 진행되고 있다. OLED는 음극에서 공급 되는 전자와 양극에서 공급되는 정공이 결합해서 가시광선 으로 변환되는 발광원리에 기반하고 있는데, 빛이 고굴절률 의 유기층 내에서 만들어지기 때문에 내부전반사 현상과 음 극의 흡수나 표면 플라스몬(surface plasmon)에 의한 손실로 인해 상당히 많은 빛의 손실이 발생한다. 따라서 이러한 손 실을 줄이고 더 많은 빛을 외부로 방출시키기 위한 광추출효 율(Outcoupling Efficiency, OCE) 향상에 관한 연구가 활발하 게 진행되어 왔다.

OLED 내 발생한 빛은 전자수송층(Electron Transport Layer, ETL)이나 정공수송층(Hole Transport Layer, HTL) 등의 유 기층 및 투명전극인 Indium tin oxide(ITO) 내에 갇히는 도파 관 모드(Waveguide mode)와 유리기판 내에 갇히는 기판 모 드(Substrate mode), 그리고 금속 재질의 음극에 의한 흡수와 표면 플라스몬의 여기에 의한 손실 등을 통해 상당 부분이 외부로 빠져나가지 못하고 내부에서 손실되어 버린다. 따라 서 $\mathrm{OCE}$ 를 향상시키기 위한 방법으로는 마이크로렌즈 배열 이나 확산층을 활용해 기판모드를 줄이는 방법 ${ }^{[5-12]}, \mathrm{OLED}$ 의 내부 구조를 다양한 구조로 변형시켜 도파관모드에 갇힌 빛 을 추출하는 방법 ${ }^{[13-20]}$, 그리고 음극에 의한 손실을 줄이는 방법 ${ }^{[21]}$ 및 이러한 방법들을 혼합한 하이브리드 방법 ${ }^{[22-23]}$ 으 로 구분할 수 있다. 최근 OLED 내 발광자의 방향, 즉 쌍극 자의 진동방향에 따라 OLED의 효율이 큰 폭으로 달라질 수 있다는 연구 결과들이 발표된 바 있다. ${ }^{[24-29]}$ 수직 진동자의 경우 형성된 복사파의 대부분이 측면을 향해 전파되면서 도 파관모드로 갇히거나 표면 플라스몬의 여기에 기여하면서 손실되어 버리지만, 수평 진동자의 경우에는 유리기판 방향 으로 방출되는 빛의 비중이 커서 OLED의 효율 향상에 기여 할 수 있다. 우리는 선행 시뮬레이션 연구를 통해 발광자(쌍 극자)의 진동방향에 따라 $\mathrm{OLED}$ 의 효율이 어떻게 달라지고 여기에 광결정(Photonic Crystal)을 적용했을 때 OCE가 얼마 나 상승할 수 있는지 보고한 바 있다. ${ }^{[30]}$ 본 연구에서는 유리 기판 속에 산란입자를 넣고 기판의 표면에 렌즈 형상을 입혔 을 때 $\mathrm{OCE}$ 가 어떻게 향상되는지를 집중적으로 연구하였다. 특히 수평방향 진동쌍극자를 가진 OLED의 OCE가 수직방 향 진동쌍극자로 설정된 OLED에 비해 훨씬 좋았던 이전 결 과를 바탕으로 이번 연구에서는 수평방향 진동쌍극자로 광 원이 설정된 OLED의 유리기판에 산란입자와 렌즈를 적용하 였을 때 나타나는 효과를 집중적으로 분석하고자 하였다. 본 연구의 결과를 등방적 광원을 설정했던 기존 연구 결과 ${ }^{[11]}$ 와 비교함으로써 발광자의 진동방향이 $\mathrm{OCE}$ 에 미치는 영향을 체계적으로 분석할 수 있을 것이다.

\section{II. 시뮬레이션 조건}

본 연구에서는 배면발광형(bottom-emission type) OLED의 $\mathrm{OCE}$ 에 대해 조사하였다. 우선 시간영역유한차분법(Finite Difference Time Domain method, FDTD method)을 이용해 $\mathrm{OLED}$ 의 기본 구조를 그림 1 과 같이 구성하였다. 사용된 소 프트웨어는 FDTD Solutions(Lumerical Solutions, Vancouver, Canada)였다. 음극은 $100 \mathrm{~nm}$ 두께의 알루미늄 재질로 구성 되었고, $\mathrm{ETL}$ 과 $\mathrm{HTL}$ 의 굴절률은 각각 1.66 과 1.82 로 설정되 었으며 투명전극(ITO)은 $150 \mathrm{~nm}$ 의 두께에 1.86 의 굴절률을 가지도록 설정하였다.[3] 투명전극 위에 위치한 유리기판의 굴절률은 1.48 로 설정하였다. ETL과 HTL의 경계면의 정가 운데에 진동하는 쌍극자를 광원으로 배치하였다. 쌍극자의 방향은 계면에 나란한 것(horizontal emitter)과 수직인 것(vertical emitter) 등 두 가지 방향으로 설정하였고 $550 \mathrm{~nm}$ 의 단색광 을 이용하였다. 이 파장은 대략적으로 가시광선의 중심 파장 을 대표한다고 볼 수 있고 사람 눈의 시감도 곡선이 최대가 되는 파장과 거의 동일하다. 아울러 본 연구에서 $\mathrm{OCE}$ 향상 을 위해 활용할 Mie 산란(Mie scattering) 현상은 파장에 대 한 의존성이 레일레이 산란에 비해 현저히 작다는 것이 알려 져 있으므로 단색광으로 진행한 시뮬레이션 결과를 일반화 시켜 적용할 수 있을 것으로 판단된다. FDTD로 조사한 OLED 의 시뮬레이션 면적은 $10 \times 10 \mu \mathrm{m}^{2}$ 였다. 투명전극과 유리기판 의 계면에서 기판 쪽으로 $95 \mathrm{~nm}$ 떨어진 곳에 검출기를 설치 하여 OLED의 유기층/ITO에서 유리기판으로 올라오는 광량 과 광도 분포를 측정한 후에 이 광선 데이터(Ray data)를 광 선추적 시뮬레이션 소프트웨어인 ASAP(Breault Research Organization, Tucson, USA)으로 가지고 온 후에 유리 기판 에서 공기로 빠져나가는 광량과 광도분포를 조사하였다. $\mathrm{ASAP}$ 에서 구축한 $\mathrm{OLED}$ 의 면적과 두께는 각각 $2 \times 2 \mathrm{~mm}^{2}$ 과 $0.7 \mathrm{~mm}$ 였고 FDTD에서 구한 ray data는 유리 기판의 바닥 으로부터 $17 \mu \mathrm{m}$ 떨어진 곳에 $0.5 \times 0.5 \mathrm{~mm}^{2}$ 의 면적을 가진 가상의 면광원의 출광데이터로 활용되었다.

이러한 기본 구조 위에 세 가지 종류의 광추출구조를 조사 하였다. 첫 번째로는 $\mathrm{SiN}($ 굴절률=1.9) 원통으로 구성된 광결

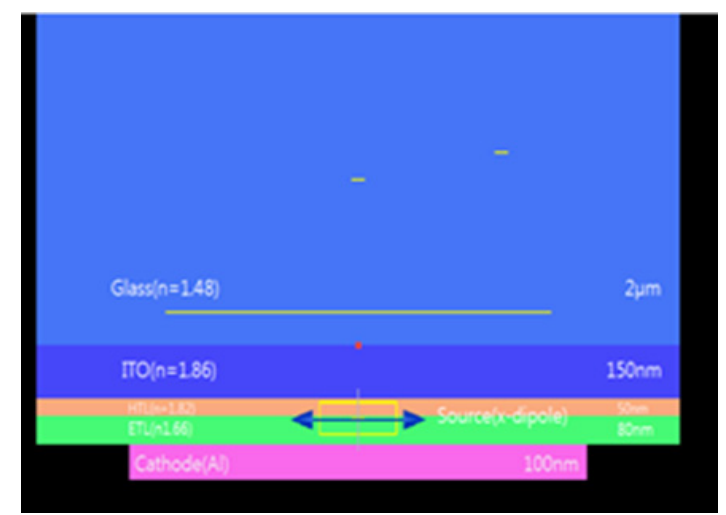

FIG. 1. The cross-section of the bottom-emission type OLED investigated in this study. 


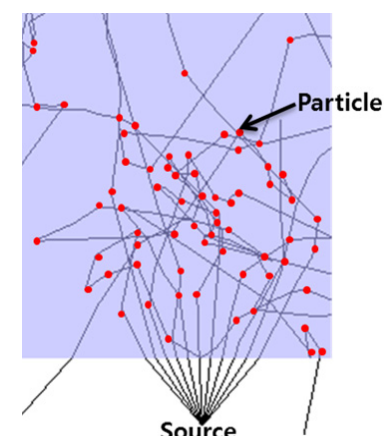

(a)
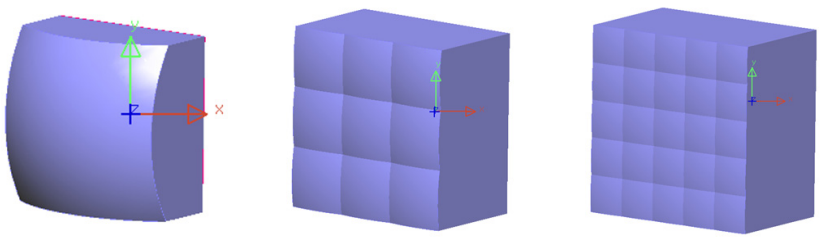

(b)

FIG. 2. (a) Mie scattering particles randomly dispersed in the glass substrate and the light path ways. (b) Pillow lenses formed on the front surface of the glass substrate.

정 층을 ITO와 유리기판 사이에 삽입한 구조로써, 이 구조의 OCE 성능에 대해서는 이미 선행연구 ${ }^{[30,}{ }^{32]}$ 를 통해 충분히 보고된 바 있다. 이 연구에 따르면 정사각형 격자 형태로 배 치된 광결정의 최적구조는 주기가 $500 \mathrm{~nm}$ 이었고, 반지름은 수평진동 쌍극자와 수직진동 쌍극자에 대해 각각 $130 \mathrm{~nm}$ 와 $200 \mathrm{~nm}$ 였다. 광결정의 높이는 OCE에 큰 영향을 주지 않았 다. 본 연구에서는 광결정 구조에 더해 유리기판 속에 100 600 나노미터 지름의 Mie 산란 입자를 넣고 유리기판의 표 면에 소위 필로우렌즈(Pillow lens)를 1개에서 25개까지 형성 한 후 이들이 각 쌍극자 광원 별로 어느 정도로 $\mathrm{OCE}$ 을 향상 시키는지 조사하였다. 그림 2(a)는 Mie 산란입자가 포함된 유리 기판 속을 진행하는 빛의 경로를 보여주고 있고, 그림 2(b)는 본 연구에 사용된 Pillow 렌즈의 형상을 보여준다. Pillow 렌즈의 곡면은 구면의 일부인데, 밑면의 형상이 사각 형이라 유리기판의 전체면적을 남김없이 덮을 수 있다는 장 점이 있다. 본 연구에서는 동일 면적상에서 렌즈의 개수, 반 경에 따라 $1 \times 1,3 \times 3,5 \times 5$ 의 3 가지 구조를 설계하였다.

\section{III. 결과 및 논의}

기존의 선행연구 ${ }^{[30]}$ 에 의하면 수평진동과 수직진동 쌍극자 광원이 포함된 $\mathrm{OLED}$ 의 $\mathrm{OCE}$ 은 $\mathrm{ETL}$ 두께가 각각 $100 \mathrm{~nm}$ 과 $180 \mathrm{~nm}$ 인 조건에서 약 $43 \%$ 와 $10 \%$ 의 $\mathrm{OCE}$ 를 보였다. 여기 에 광결정구조를 형성할 경우 이 효율은 $54 \%$ 와 $13 \%$ 정도로 향상되었다. 그림 3은 광결정이 적용된 OLED로부터 공기로 출광된 빛의 광도 분포를 수평진동 쌍극자 조건에 대해 구한 것이다. 광결정을 구성하는 $\mathrm{SiN}$ 실린더가 정사각형 격자 형 태로 배치되어 있기 때문에 광도 분포에서도 4 축대칭성이
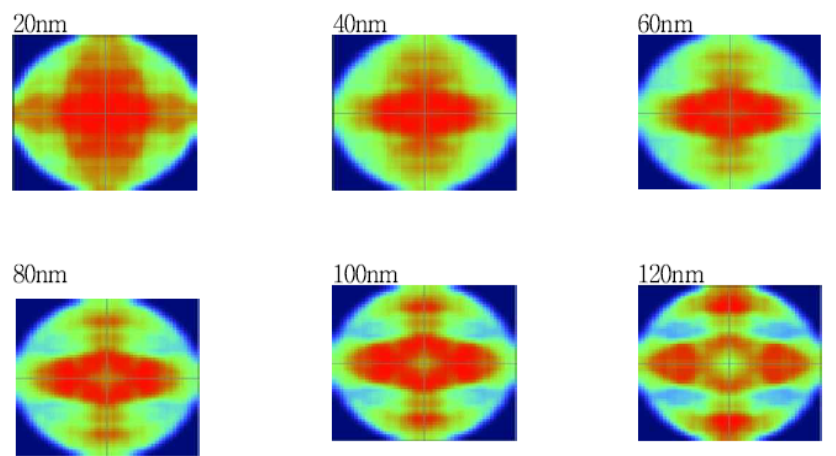

FIG. 3. Far-field intensity distribution proportional to $|E|^{2}$, where $E$ is the electric field, detected outside of OLED as a function of the ETL thickness for a horizontal emitter condition.

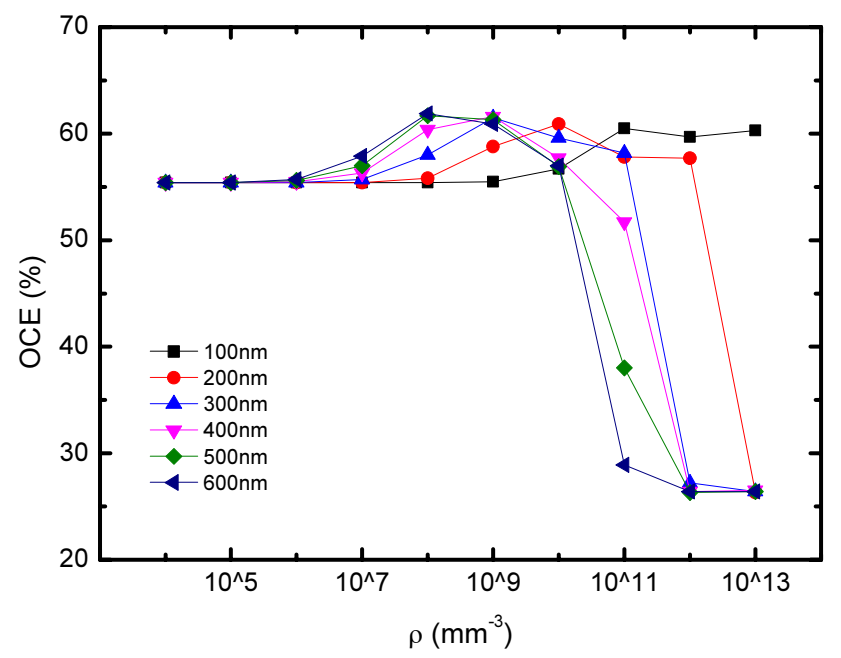

FIG. 4. The dependence of the OCE on the density and the radius of the Mie scatterers embedded in the glass substrate at the ETL thickness of $100 \mathrm{~nm}$.

뚜렷이 보임을 알 수 있다. 이러한 특징은 각도에 따라 밝기 나 색상이 현저하게 변할 수 있다는 것을 의미하고 있고, 따 라서 광결정을 OLED에 적용하는데 있어서 주의가 필요하다 는 것을 보여준다. 이러한 단점은 몇 가지 방법으로 보완될 수 있는데, 가령 무작위적인 방향성을 가지고 있는 회절격자 구조를 형성하거나 ${ }^{[16]}$ 아래에서 소개할 $\mathrm{Mie}$ 산란입자를 활용 하는 것이 몇 가지 예가 될 것이다.

그림 4는 광결정의 최적구조와 ETL의 최적 두께 $(100 \mathrm{~nm})$ 가 적용된 $\mathrm{OLED}$ 의 유리기판 내에 $\mathrm{Mie}$ 산란입자를 넣었을 경우 입자의 밀도와 지름에 따라 OCE가 어떻게 변하는지를 보여준다. 입자의 지름에 따라 달라지지만 대략적으로 입자 의 밀도가 $10^{6} / \mathrm{mm}^{3}$ 을 넘어서게 되면 기판 모드로 갇힌 빛이 부분적으로 추출되면서 OCE가 증가함을 알 수 있다. 그렇지 만 입자의 수가 과도하게 많아지면 오히려 너무 많은 충돌이 일어나 빛의 진행을 방해하게 되어 OCE가 큰 폭으로 떨어 지게 된다. 단, 입자의 지름이 $100 \mathrm{~nm}$ 인 경우에는 체적이 상 대적으로 적어서 $10^{13} / \mathrm{mm}^{3}$ 의 밀도에서도 높은 $\mathrm{OCE}$ 가 유지 
되고 있음을 알 수 있다. Mie 산란입자의 직경이 $600 \mathrm{~nm}$, 밀 도가 $10^{8} / \mathrm{mm}^{3}$ 일 때 $\mathrm{OCE}$ 는 약 $62 \%$ 였다. 그림 4 의 결과는 $\mathrm{Mie}$ 산란입자의 직경이 사용된 광원의 파장인 $550 \mathrm{~nm}$ 와 비 슷한 경우(즉, 400 600 nm 범위인 경우) 밀도가 $10^{7} \sim 10^{9} / \mathrm{mm}^{3}$ 일 때 거의 동일한 $\mathrm{OCE}$ 를 얻을 수 있음을 보여준다. 이는 $\mathrm{Mie}$ 산란 효율이 사용된 광원 및 산란입자의 직경 사이의 상관성과 관련되어 있음을 보여주는 것이다.

그림 5는 Pillow 렌즈가 유리기판의 전면에 형성되었을 경

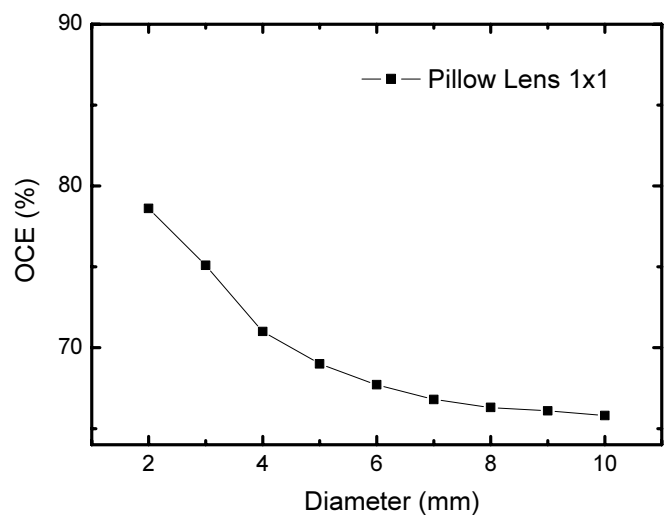

(a)

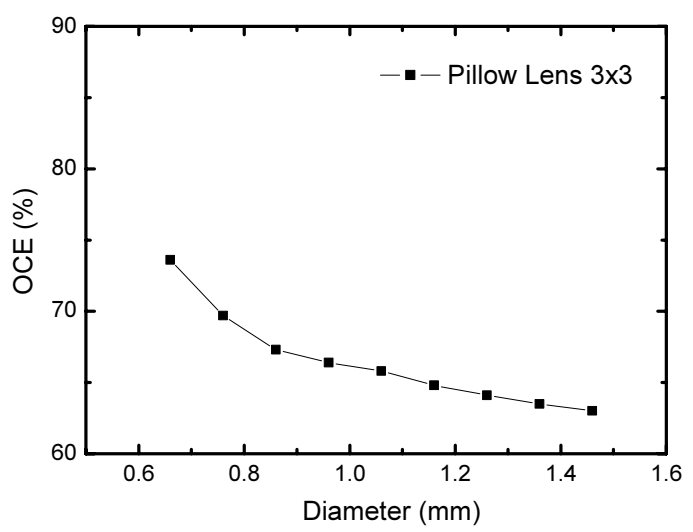

(b)

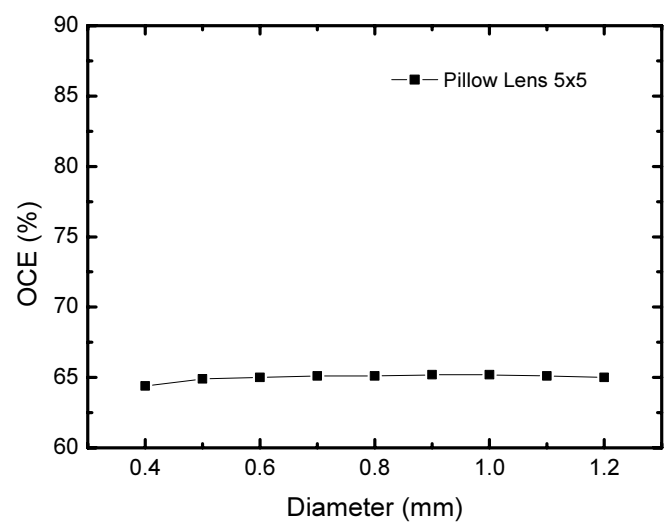

(c)

FIG. 5. OCE as a function of the diameter for (a) $1 \times 1$, (b) $3 \times 3$ and (c) $5 \times 5$ pillow lens arrays. The particle density and the diameter were fixed to be $10^{8} \mathrm{~mm}^{-3}$ and $600 \mathrm{~nm}$, respectively.
우 렌즈의 개수와 곡률지름에 따라 OCE가 어떻게 변하는지 보여준다. OCE는 Pillow 렌즈의 개수가 1 개일 때 $2 \mathrm{~mm}$ 지 름을 가진 렌즈에 대해 가장 높은 수치( $77 \%)$ 를 보였다. 유 리기판의 측면 길이가 $2 \mathrm{~mm}$ 이므로 이 단일 렌즈에 대해서 는 구형 렌즈의 중심 근처에 광원이 놓여지게 되는 효과를 가져오고 따라서 기판-공기의 계면에서 전반사가 거의 일어 나지 않게 된다. Pillow 렌즈의 곡률지름이 커지면 렌즈의 표 면이 평면에 더 가까워지게 되므로 $\mathrm{OCE}$ 는 감소한다. 렌즈의 개수가 9 개로 늘어나게 되면 구면렌즈의 위치와 광원 위치 사이의 상관성이 줄어들면서 기판-공기 계면에서 발생하는 전반사를 완전히 없앨 수 없어서 단일 렌즈로 구성된 OLED 에 비해 OCE가 저하하게 된다. 렌즈의 개수가 25 개로 늘어 나는 경우에는 $\mathrm{OCE}$ 가 $54 \%$ 에서 약 $65 \%$ 정도로 늘어나기는 하지만 $\mathrm{OCE}$ 의 렌즈 지름에 대한 의존성은 존재하지 않는다. 이는 발광원에서 나온 빛을 전반사시키지 않고 굴절, 추출시 키는 능력이 주어진 렌즈의 곡률 범위 내에서는 거의 동일하 다는 것을 의미한다.

이상의 결과를 토대로 얻어진 최적 조건, 즉 $\mathrm{Mie}$ 산란입자 의 최적 지름과 밀도, Pillow 렌즈의 최적 개수와 곡률지름을 광결정 구조가 포함된 OLED에 적용한 후 ETL의 두께 변화 에 따른 $\mathrm{OCE}$ 와 광도 분포를 확인하였다. 그림 6은 ETL의 두께에 따른 광도분포를 보여준다. 이 결과는 그림 3 에서 보 였던 4축 대칭성이 상당히 완화되면서, 특히 ETL의 두께가 작은 영역에서 비교적 균일한 광도분포를 나타낸다는 점을 보여준다. 이는 기본적으로 유리기판 속에 포함된 Mie 산란 입자들의 산란 효과가 기판 아래에 위치한 광결정 구조가 형 성한 대칭적 광도분포를 흐트러뜨린다는 것을 의미한다. 따 라서, 본 결과는 확산층을 OLED 내에 도입하게 되면 광결 정 구조에 의해서 형성되는 각도에 따른 광도 분포와 색분산 의 문제점을 어느 정도 해결할 수 있음을 보여준다.

그림 7은 세 가지 광추출구조를 적용한 수평진동 쌍극자형 $\mathrm{OLED}$ 의 ETL 두께에 따른 $\mathrm{OCE}$ 를 보여주고 있다. 지금까지 의 모든 결과는 수평진동 쌍극자를 포함한 OLED에 대한 것 이었는데, 모든 시뮬레이션 과정을 광원이 수직진동 쌍극자
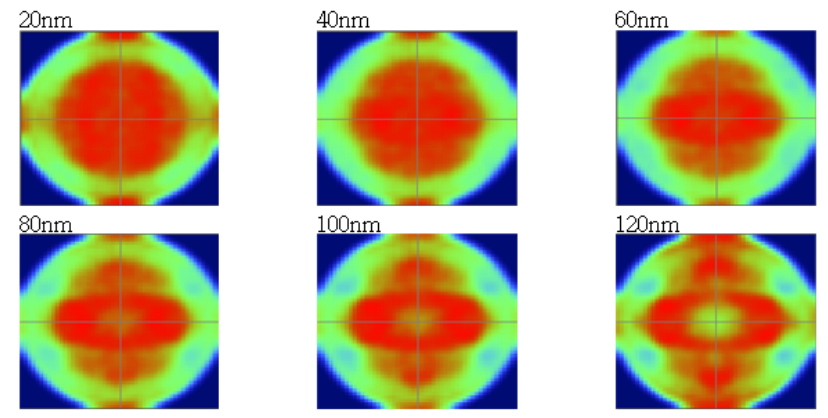

FIG. 6. Far-field intensity distribution proportional to $|E|^{2}$, where $E$ is the electric field, detected outside of OLED as a function of the ETL thickness for a horizontal emitter condition. The optimized conditions of the Mie scattering particles and the pillow lens were applied to the OLED. 


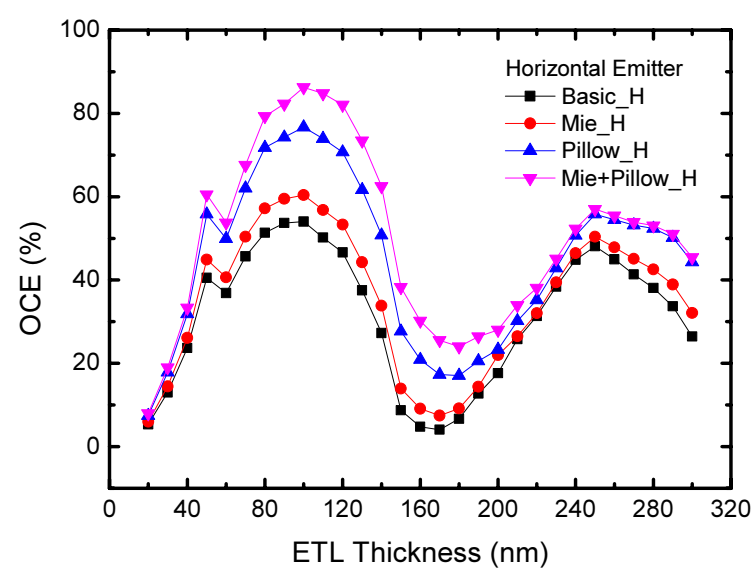

(a)

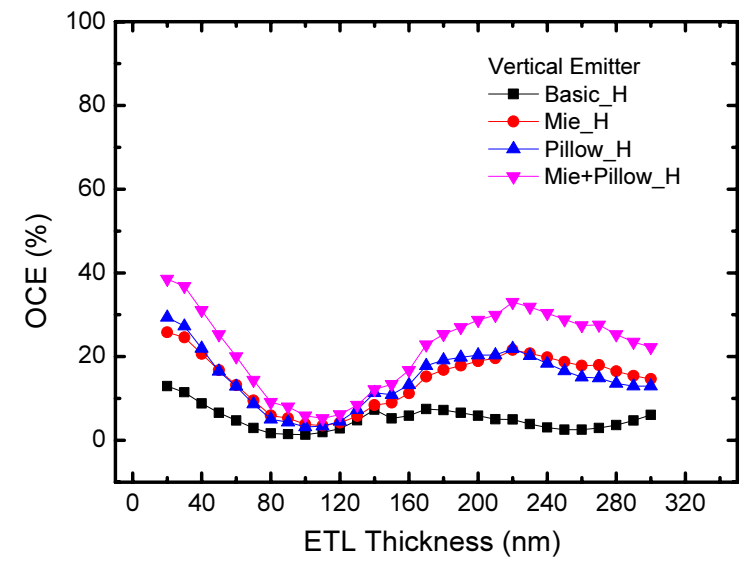

(b)

FIG. 7. The dependence of OCE on the ETL thickness obtained under various conditions for (a) the horizontal emitter and (b) the vertical emitter. "Basic" indicates no outcoupling structure except for the photonic crystal layer is applied to OLED. For other three conditions, the optimized photonic crystal layer is also adopted.

로 설정된 $\mathrm{OLED}$ 에 대해서도 적용해 동일한 조건 하에서 $\mathrm{OCE}$ 를 구할 수 있고, 그 결과가 그림 7(b)에 제시되어 있다. 이 비교로부터 세 가지 결론을 내릴 수 있다. 우선 광원으로 설정된 쌍극자의 진동 방향에 따라 $\mathrm{OCE}$ 가 현저하게 달라진 다. 전체적으로 수평진동 쌍극자의 경우가 수직진동 쌍극자 의 경우에 비해 훨씬 높은 $\mathrm{OCE}$ 를 보여준다. 이는 쌍극자의 진동에 따라 형성된 전자기파의 진행 방향 및 음극 표면에서 의 표면 플라스몬의 여기 정도가 쌍극자의 진동 방향에 크게 의존한다는 것을 의미한다. 본 연구의 결과와 기존 결과 ${ }^{[31]}$ 을 비교해 보면 본 연구에서 얻어진 수평진동 쌍극자와 수직진 동 쌍극자의 결과를 고려해 등방적인 광원의 결과로 변환하 면 기존 등방적인 광원이 적용된 결과 ${ }^{[31]}$ 와 동일한 결론이 얻어짐을 알 수 있고 이는 본 시뮬레이션의 결론이 타당함을 보여준다고 생각된다. 둘째, 쌍극자의 진동 방향에 따라 OCE의 진동에서 보이는 간섭 효과가 반대로 나타난다. 수평 진동 쌍극자의 경우에는 ETL 두께 약 $100 \mathrm{~nm}$ 근처에서 OCE가 최대를 이루지만 수직진동 쌍극자의 경우에는 비슷
한 $\mathrm{ETL}$ 두께에서 $\mathrm{OCE}$ 가 최소가 된다. 이러한 결과는 진동 자가 발생하는 전자기파 중 유리기판으로 향하는 빛과 금속 을 향하는 빛의 초기 위상이 다르기 때문이다. ${ }^{[25]}$ 이 초기 위 상이 수평진동 쌍극자의 경우에는 거의 동일하지만 수직진 동 쌍극자의 경우에는 $\pi$ 만큼의 차이를 보인다. 마지막으로, 최적화된 Mie 산란 입자와 Pillow 렌즈를 수평진동 쌍극자가 포함된 OLED에 적용할 경우 $\mathrm{OCE}$ 가 매우 큰 폭으로 증가함 을 알 수 있다. 광결정 구조만 적용된 경우 54\%였던 OCE가 세 가지 구조를 모두 적용한 경우에는 $86 \%$ 까지 약 1.6 배 증 가함을 알 수 있다. 특히 산란 입자의 적용은 광결정구조가 만들어 내는 독특한 광도분포를 완화시켜서 조명에 적합한 부드러운 광도분포를 구현할 수 있다는 면에서 중요성을 가 진다고 하겠다.

마지막으로 본 연구 결과를 적용하는데 있어서 발생하는 문제점 혹은 한계를 정리하고자 한다. 본 연구에서 제안한 것과 같이 유리 기판에 산란입자를 포함시키는 것은 제작상 의 문제 및 외부광의 산란 반사에 의한 콘트라스트 저하 등 의 문제가 발생하므로 OLED 디스플레이 적용하는 것은 현 실성이 없을 것으로 보인다. 따라서 본 연구 결과는 조명용 백색 $\mathrm{OLED}$ 의 효율을 높이기 위한 광학구조를 제안한 것으 로 그 의미를 한정하여야 한다. 아울러 유리에 산란입자를 포함시키는 제작상의 문제를 극복하기 위해서는 유리와 유 기층의 사이에 별도의 산란층을 삽입하는 방법이 하나의 대 안이 될 수 있을 것이다.

\section{IV. 결 론}

본 연구에서는 광결정 구조가 포함된 OLED에 대해 광원 으로 설정된 쌍극자의 진동 방향, 유리기판에 적용된 Mie 산 란입자와 Pillow 렌즈가 광추출효율에 대해 미치는 영향을 FDTD와 광선추적기법을 결합한 시뮬레이션을 통해 분석하 였다. 쌍극자의 진동 방향이 수평인 경우 $\mathrm{Mie}$ 산란입자의 최 적 조건은 직경 $600 \mathrm{~nm}$, 밀도 $10^{8} / \mathrm{mm}^{3}$ 였고 이 때의 $\mathrm{OCE}$ 는 약 $60 \%$ 였다. Pillow 렌즈가 적용되었을 경우 $\mathrm{OCE}$ 는 렌즈의 개수가 1 개이고 $2 \mathrm{~mm}$ 지름을 가진 렌즈에 대해 가장 높은 수치( $77 \%)$ 를 보였다. 수평진동 쌍극자가 광원으로 설정된 OLED의 ETL의 두께가 약 $100 \mathrm{~nm}$ 일 때 OCE가 최대가 되 었고 Mie 산란입자, Pillow 렌즈, 그리고 이 두 구조를 한꺼 번에 적용한 OLED 순으로 $\mathrm{OCE}$ 가 향상되었고 최대 OCE는 약 $86 \%$ 였다. 반면에 쌍극자의 진동방향이 수직인 경우에는 모든 조건을 다 적용한 경우에도 $\mathrm{OCE}$ 가 $40 \%$ 를 넘지 못하였 다. 이러한 결과는 OLED 내 발광 분자의 배향을 조절하고 유기층의 두께 및 광추출 구조를 최적화할 경우 OLED의 효 율을 크게 향상시킬 수 있다는 점을 보여준다.

\section{References}

1. K. Hong and J.-L. Lee, "Recent developments in light extraction technologies of organic light emitting diodes," 
Electron. Mater. Lett. 7, 77-91 (2011).

2. W. Brütting, J. Frischeisen, T. D. Schmidt, B. J. Scholz, and C. Mayr, "Device efficiency of organic light-emitting diodes: Progress by improved light outcoupling," Phys. Status Solidi A 210, 44-65 (2012).

3. G. Gu, D. Z. Garbuzov, P. E. Burrows, S. Venkatesh, and S. R. Forrest, "High-external-quantum-efficiency organic light-emitting devices," Opt. Lett. 22, 396-398 (1997).

4. J. Frischeisen, B. Scholz, B. Arndt, T. Schmidt, R. Gehlhaar, C. Adachi, and W. Brütting, "Strategies for enhanced light extraction from surface plasmons in organic light-emitting diodes," J. Photonic. Energy 1, 011004 (2011).

5. C. F. Madigan, M.-H. Lu, and J. C. Sturm, "Improvement of output coupling efficiency of organic light-emitting diodes by backside substrate modification," Appl. Phys. Lett. 76, 1650-1652 (2000)

6. S. Moller and S. R. Forrest, "Improved light out-coupling in organic light emitting diodes employing ordered microlens arrays," J. Appl. Phys. 91, 3324-3327 (2002).

7. M.-L. Chen, A.-C. Wei, and H.-P. Shieh, "Increased organic light-emitting diode panel light efficiency by optimizing structure and improving alignment of pyramidal array lightenhancing layers,” Jpn. J. Appl. Phys. 46, 1521-1525 (2007).

8. C.-J. Yang, S.-H. Liu, H.-H. Hsieh, C.-C. Liu, T.-Y. Cho, and C.-C. $\mathrm{Wu}$, "Microcavity top-emitting organic light-emitting devices integrated with microlens arrays: Simultaneous enhancement of quantum efficiency, cd/A efficiency, color performances, and image resolution,” Appl. Phys. Lett. 91, 253508 (2007).

9. Y.-H. Cheng, J.-L. Wu, C.-H. Cheng, K.-C. Syao, and M.-C. M. Lee, "Enhanced light outcoupling in a thin film by texturing meshed surfaces," Appl. Phys. Lett. 90, 091102 (2007).

10. C.-C. Liu, S.-H. Liu, K.-C. Tien, M.-H. Hsu, H.-W. Chang, C.-K. Chang, C.-J. Yang, and C.-C. Wu, "Microcavity top-emitting organic light-emitting devices integrated with diffusers for simultaneous enhancement of efficiencies and viewing characteristics," Appl. Phys. Lett. 94, 103302 (2009).

11. N. Nakamura, N. Fukumoto, F. Sinapi, N. Wada, Y. Aoki, and K. Maeda, "Glass substrates for OLED lighting with high out-coupling efficiency,” SID’09 Tech. Digest, 603-606 (2009).

12. S. S. Jeong and J.-H. Ko, "Simulation study on the optical structures for improving outcoupling efficiency of organic light emitting diodes,” J. Inf. Disp. 13, 139-143 (2012).

13. S. Okutani, N. Kamiura, H. Sano, T. Sawatani, D. Fujita, T. Takehara, K. Sunohara, and M. Kobayashi, "A 20.8-inch WXGA full color AMOLED display by integrating scattering reflector with micro-bumps," SID'07 Tech. Digest, 173-176 (2007).

14. Y. Sun and S. R. Forrest, "Enhanced light out-coupling of organic light-emitting devices using embedded low-index grids," Nature Photonics 2, 483-487 (2008).

15. T.-W. Koh, J.-M. Choi, S. Lee, and S. Yoo, “Optical outcoupling enhancement in organic light-emitting diodes: Highly conductive polymer as a low-index layer on microstructured ITO electrodes," Adv. Mater. 22, 1849-1853 (2010).

16. W. H. Koo, S. M. Jeong, F. Araoka, K. Ishikawa, S. Nishimura, T. Toyooka, and H. Takezoe, "Light extraction from organic light-emitting diodes enhanced by spontaneously formed buckles," Nature Photonics 4, 222-226 (2010).

17. Y.-J. Lee, S.-H. Kim, J. Huh, G.-H. Kim, Y.-H. Lee, S.-H. Cho, Y.-C. Kim. and Y. R. Do, "A high-extraction-efficiency nanopatterned organic light-emitting diode," Appl. Phys. Lett. 82, 3779-3781 (2003).

18. T. Tsutsui, M. Yahiro, H. Yokogawa, K. Kawano, and M. Yokoyama, "Doubling coupling-out efficiency in organic light-emitting devices using a thin silica aerogel layer," Adv. Mater. 13, 1149-1152 (2001).

19. H. J. Peng, Y. L. Ho, X. J. Yu, and H. S. Kwok, "Enhanced coupling of light from organic light emitting diodes using nanoporous films," J. Appl. Phys. 96, 1649-1654 (2004).

20. K. Hong, H. K. Yu, I. Lee, K. Kim, S. Kim, and J.-L. Lee, "Enhanced light out-coupling of organic light-emitting diodes: Spontaneously formed nanofacet-structured $\mathrm{MgO}$ as a refractive index modulation layer," Adv. Mater. 22, 4890-4894 (2010).

21. J.-B. Kim, J.-H. Lee, C.-K. Moon, S.-Y. Kim, and J.-H. Kim, "Highly enhanced light extraction from surface plasmonic loss minimized organic light-emitting diodes," Adv. Mater. 25, 3571-3577 (2013).

22. J.-H. Jang, K.-J. Kim, J.-H. Kim, and M.-C. Oh, "Outcoupling enhancement of OLED using microlens array and diffractive grating," Korean J. Opt. Photon. (Hankook Kwanghak Hoeji) 18, 441-446 (2007).

23. S. Reineke, F. Lindner, G. Schwartz, N. Seidler, K. Walzer, B. Lüssem, and K. Leo, "White organic light-emitting diodes with fluorescent tube efficiency," Nature (London) 459, 234-239 (2009).

24. J.-S. Kim, P. K. H. Ho, N. C. Greenham, and R. H. Friend, "Electroluminescence emission pattern of organic lightemitting diodes: Implications for device efficiency calculations," J. Appl. Phys. 88, 1073-1081 (2000).

25. M. Flämmich, M. C. Gather, N. Danz, D. Michaelis, A. H. Bräuer, K. Meerholz, and A. Tünnermann, "Orientation of emissive dipole in OLEDs: Quantitative in situ analysis," Org. Electron. 11, 1039-1046 (2010).

26. M. Flämmich, J. Frischeisen, D. S. Setz, D. Michaelis, B. C. Krummacher, T. D. Schmidt, W. Brütting, and N. Danz, "Oriented phosphorescent emitters boost OLED efficiency," Org. Electron. 12, 1663-1668 (2011).

27. P. Liehm, C. Murawski, M. Furno, B. Lüssem, K. Leo, and M. C. Gather, "Comparing the emissive dipole orientation of two similar phosphorescent green emitter molecules in highly efficient organic light-emitting diodes," Appl. Phys. Lett. 101, 253304 (2012).

28. S.-Y. Kim, W.-I. Jeong, C. Mayr, Y.-S. Park, K.-H. Kim, J.-H. Lee, C.-K. Moon, W. Brütting, and J.-J. Kim, "Organic light-emitting diodes with $30 \%$ external quantum effi ciency 
based on a horizontally oriented emitter," Adv. Funct. Mater. 23, 3896-3900 (2013).

29. K.-H. Kim, C.-K. Moon, J.-H. Lee, S.-Y. Kim, and J.-J. Kim, "Highly efficient organic light-emitting diodes with phosphorescent emitters having high quantum yield and horizontal orientation of transition dipole moments," Adv. Mater. DOI: 10.1002/adma.201305733 (2014).

30. J. S. Lee, J.-H. Ko, J. Park, and J. W. Lee, "Simulation study on the effect of the emitter orientation and photonic crystals on the outcoupling efficiency of organic light- emitting diodes," submitted to J. Opt. Soc. Korea.

31. S. S. Jeong and J.-H. Ko, "Optical simulation study on the effect of diffusing substrate and pillow lenses on the outcoupling efficiency of organic light emitting diodes," J. Opt. Soc. Korea 17, 269-274 (2013).

32. S. S. Jeong, H.-W. Choi, and J.-H. Ko, "Simulation study on the outcoupling efficiency and intensity distribution of photonic crystal-based organic light-emitting diodes," New Physics: Sae Mulli 63, $892-899$ (2013). 